\title{
Modeling the functional state of the reverse transcriptase of hepatitis $B$ virus and its application to probing drug-protein interaction
}

Xiaojun Xu', Hong Thai ${ }^{1}$, Kathryn M. Kitrinos², Guoliang Xia', Anuj Gaggar², Matthew Paulson², Lilia Ganova-Raeva', Yury Khudyakov ${ }^{1}$ and James Lara ${ }^{1 *}$

\begin{abstract}
Background: Herein, the predicted atomic structures of five representative sequence variants of the reverse transcriptase protein (RT) of hepatitis B virus (HBV), sampled from patients with rapid or slow response to tenofovir disoproxil fumarate (TDF) treatment, have been examined to identify structural variations between them in order to assess structural and functional properties of HBV-RT variants associated with the differential responses to TDF treatment.

Results: We utilized a hybrid computational approach to model the atomistic structures of HBV-RT/DNA-RNA/dATP and HBV-RT/DNA-RNA/TFV-DP (tenofovir diphosphate) complexes with the native hybrid DNA-RNA substrate in place. Multi-nanosecond molecular dynamics (MD) simulations of HBV-RT/DNA-RNA/dATP complexes revealed strong coupling of the natural nucleotide substrate, dATP, to the active site of the RT, and the differential involvement of the two putative magnesium cations $\left(\mathrm{Mg}^{2+}\right)$ at the active site, whereby one $\mathrm{Mg}^{2+}$ directly bridges the interaction between dATP and HBV-RT and the other serves as a coordinator to maintain an optimal configuration of the active site. Solvated interaction energy (SIE) calculated in MD simulations of HBV-RT/DNA-RNA/TFV-DP complexes indicate no differential binding affinity between TFV-DP and HBV-RT variants identified in patients with slow or rapid response to TDF treatment.

Conclusion: The predicted atomic structures accurately represent functional states of HBV-RT. The equivalent interaction between TFV-DP and each examined HBV-RT variants suggests that binding affinity of TFV-DP to HBV-RT is not associated with delayed viral clearance.
\end{abstract}

Keywords: Hybrid structure modeling, Molecular dynamics (MD), Solvated interaction energy (SIE), Hepatitis B, Tenofovir, Drug resistance, Reverse transcriptase

\section{Background}

During the life cycle of HBV, RT utilizes a single-stranded viral genomic RNA as a template to synthesize a hybrid RNA-DNA duplex, and then converts it to doublestranded DNA (ds-DNA). As this step is critical for the viral genome replication, it makes RT an attractive target for antiviral treatment. Thus, both nucleoside and nucleotide RT inhibitors (NRTIs) and non-nucleoside RT

\footnotetext{
* Correspondence: xz15@cdc.gov

'Division of Viral Hepatitis, National Center for HIV, Hepatitis, STD and TB Prevention, Centers for Disease Control and Prevention, Atlanta, GA 30333, USA

Full list of author information is available at the end of the article
}

inhibitors (NNRTI) are extensively used as antiretroviral agents against HBV and HIV infection [1, 2].

The effective HBV NRTIs are nucleoside or nucleotide analogs that can be phosphorylated to their diphosphate or triphosphate active forms by intracellular kinases. Active forms of NRTIs incorporate into the elongating DNA strand, terminate the elongation of the nascent DNA strand, and prevent additional dNTPs from being incorporated. Tenofovir disoproxil fumarate (TDF) is an oral prodrug of nucleotide analog tenofovir (TFV, PMPA, 9[(R)-2-(phosphonomethoxy)propyl]adenine). TDF is rapidly 
converted to TFV following absorption and is readily catalyzed to the active diphosphate form, TFV-DP (Fig. 1) [3].

TDF is a non-selective RT inhibitor with demonstrated in vitro activity against both wild-type and mutant strains of HIV [4-6] and is also effective for treating chronic hepatitis B (CHB) [7-10]. While resistance mutations within the HBV-RT have been identified for NRTI monotherapies, such as adefovir, entecavir, lamivudine, telbivudine and clevudine [8], there is no evidence of TDF resistance [11]. However, differential therapy responses can be observed among patients and have been previously defined: a delayed viral clearance response in slow responders (SR), or a rapid decline in viral load immediately following treatment initiation in rapid responders (RR) [12, 13].

TFV resistance in the setting of HIV has been extensively studied at the molecular level [14-16]. These studies have shown that the molecular mechanism of TFV resistance is complex and involves reverse transcriptase, RNase and exonuclease activities of HIV-RT. In order to remain functional, resistant mutants of HIV-RT need to maintain their capability of incorporating nucleotide at a sufficient rate, as well as to discriminate against NRTIs or improve the excision rate of incorporated NRTIs [17].

As the HBV-RT is homologous to HIV-RT [17], it is reasonable to propose that a similar mechanism could be involved in the differential treatment response to TDF. Whether and/or how the molecular level interaction between TFV-DP and HBV-RT has a part in the aforementioned differential patient responses upon TDF treatment is unclear due to the lack of direct experimental evidence. There is no solved structure of the HBV$\mathrm{RT}$ in any form.
In this study, we set out to address the molecular level interaction between TFV-DP and HBV-RT and the relationship to patient response using a hybrid computational approach. We utilized two X-ray crystal structures of HIV-RT to model the atomic structure of HBV-RT in its functional state with the aim of delineating the detailed drug-protein interactions between TFV-DP and the active sites of genetically distinct variants of HBV-RT.

\section{Results and discussion}

Atomic structure models of the HBV-RT in its functional state

While substantial efforts have been devoted to solving the crystal structures of HIV-RT, with or without ligands (e.g. substrates, small inhibitory molecules), no experimentally derived HBV-RT structures have become available. HIV-RT and HBV-RT are recognized as homologous [17], and possess similar multiple catalytic activities. A computational approach is often applied to structural implications in drug-resistance studies on HBV-RT using HIV-RT as structural template for homology modeling of HBV-RT $[17,18]$. Still, the low sequence identity and modeling HBV RT without considering the particular functional state imposes technical difficulties in obtaining structural models of high confidence.

In models of NRTIs bound to HBV-RT, it is reasonable to hypothesize that an effective drug binds to the active site as if it were a native substrate in position to be further covalently incorporated, similar to the HIVRT/DNA-DNA/TFV-DP complex [19]. Even though we are not to rule out the possibility of a NRTI being able to bind elsewhere than the active site on HBV-RT or

a<smiles>CC(C)OC(=O)OCOP(=O)(COC(=O)OC(C)C)OCO[C@@H](C)Cn1cnc2c(N)ncnc21</smiles>

b<smiles>C[C@H](COP(=O)(O)O)Cn1cnc2c(N)ncnc21</smiles>

C<smiles>C[C@@H](COP(=O)(O)OP(=O)(O)OP(=O)(O)O)Cn1cnc2c(N)ncnc21</smiles>

Fig. 1 Chemical structures of a tenofovir disoproxil fumarate (TDF), prodrug of $\mathbf{b}$ tenofovir (TFV); $\mathbf{c}$ tenofovir diphosphate (TFV-DP) 
HIV-RT, to date there is no evidence for such type of interaction. Moreover, a putative strong binding of the drug to the active site would seem to preclude the likelihood of additional binding sites on the enzyme. To use constraints derived from experimental results to guide the refinement, we first modeled the HBV-RT structure itself using ITASSER [20, 21] with an additional template, the X-ray crystal structure of HIV-RT/ DNA-RNA/dATP [22], and then included the native substrates (the DNA-RNA duplex and dATP) from this template complex in the subsequent refinement (MD simulation).

The C-scores [20] (data not shown) of our HBV-RT models generated by ITASSER for the variants of interest indicated high confidence; however, we noticed that the exact folding patterns in the fingers domain differ (Additional file 1: Figure S1). Therefore, we chose the HBV-RT4 model (HBV-RT4, Additional file 1: Figure $\mathrm{S} 1 \mathrm{C}$ ) which has the same folding pattern as the HIV-RT template (Additional file 1: Figure S1F) in order to proceed with the refinement.

The structure of the $\mathrm{N}$-terminal 40 amino acid region was of relatively low accuracy (Additional file 1: Figure S1A-E). Thus, we re-modeled the N-terminal of HBVRT4 separately using ITASSER, and assembled it with the rest of the protein using ab initio assembly [23]. The $\mathrm{N}$-terminus in the resultant assembled structure of HBV-RT4 is of much more ordered secondary structural elements than it was in the initial model from ITASSER (Additional file 2: Figure S2).

The assembled structure was then combined with the duplex DNA-RNA and dATP from the template HIV-RT/ DNA-RNA/dATP crystal strcuture, refined by $\sim 120$ ns MD simulation in explicit solvent to obtain the final model of HBV-RT4/DNA-RNA/dATP.

After the MD simulation, the centroid of the dominant cluster (obtained by pairwise root mean square deviation, rmsd, clustering) from the second half of HBV-RT4/DNA-RNA/dATP trajectory was chosen as the representative structure for HBV-RT in its functional state (Additional file 2: Figure S2A). The corresponding part from the HIV-RT/DNA-RNA/dATP (HIV-RT residue 1 to 320) is shown in gray surface representation for comparison. Apart from the N-terminal domain partially protruding out of the template's surface envelope, all the other domains ("thumb", "fingers" and "palm" domains) are in close agreement with the template structure (Fig. 2a). The DNA-RNA duplex is clamped by the enzyme through the circular arrangement of the thumb, palm and fingers domains. Prior to entering the active site, the duplex is anchored by one $\alpha$-helix (D283 to A297) in the thumb domain. The RNA template strand is tracked along by a long loop (N123 to N131) connecting the palm and fingers domains, and captured by the fingers domain toward its 5 ' end.

In preparation for incorporation, the dATP situates itself in the active site with good base ring stacking to the penultimate dG, and by making two persistent hydrogen bonds (H-bond) with the uracil from the opposing template RNA strand (Fig. 2a, Additional file 2: Figure S2A); these features persist throughout the un-constrained MD (20 -120 ns). The triphosphate group of the dATP is stably coupled with residue L146 and D205 of HBV-RT by cooperatively chelating one $\mathrm{Mg}^{2+}$ (Fig. $2 \mathrm{~b}$ ). This particular configuration differs from the HIV-RT active site mainly by the absence of a third aspartate residue in the active site; in HIV-RT this third aspartate (D110) participates in chelating the two $\mathrm{Mg}^{2+}$ in the active site of the HIV-RT (Fig. 2c). Without this aspartate, the $\mathrm{Mg}^{2+}$ ions were stabilized over the equilibration stage of the MD by incorporating extra water molecules, and this constellation remained stable throughout in the production run (see next section).

\section{Different roles of $\mathrm{Mg}^{2+}$ ions in the active site}

Apart from the first $\mathrm{Mg}^{2+}\left(\mathrm{Mg}^{2+}-1\right.$ hereafter) being directly involved in the dATP binding to the active site, a second $\mathrm{Mg}^{2+}\left(\mathrm{Mg}^{2+}-2\right.$ hereafter $)$ is also present in the HIV-RT/DNA-RNA/dATP crystal structure. Due to an insufficiency of potential ligation atoms in the active site of HBV-RT comparing to HIV-RT, $\mathrm{Mg}^{2+}-1$ can only be coordinated by five surrounding oxygens and $\mathrm{Mg}^{2+}-2$ by only three, not considering contributions from water molecules postulated in our initial settings. In fact, water molecules filled in dynamically over the equilibration stage and stabilized the chelation as with the optimal octahedral configuration for $\mathrm{Mg}^{2+}$ over the entire $\mathrm{MD}$ simulation. To evaluate the stability of the chelation, we computed the moving average ( $0.2 \mathrm{~ns}$ window) of the distance from each non-water oxygen atom to the corresponding $\mathrm{Mg}^{2+}$ over the non-constrained MD simulation $(20-120 \mathrm{~ns})$. The result shows a persistently stable coupling of the non-water atoms to the corresponding $\mathrm{Mg}^{2+}$ ion (Fig. 3).

While it is clear that the $\mathrm{Mg}^{2+}-1$ bridges the interaction between the dATP and RT (present in the HIVRT/DNA-RNA/dATP and HIV-RT/DNA-DNA/TFV-DP crystal structure [19, 22], PDB code: 4PQU and 1 T05), the importance of the $\mathrm{Mg}^{2+}-2$ in these RTs is open to question, as it is only $\mathrm{Mg}^{2+}-1$ which appears in the HIVRT/DNA-DNA/TFV-DP structure (PDB code: 1 T05) [19]. To address this question, MD simulations with only the $\mathrm{Mg}^{2+}-1$ (i.e. by deleting the $\mathrm{Mg}^{2+}-2$ atom) were also performed for all the HBV-RT variants. On one hand, we found no significant changes in binding affinities of dATP to HBV-RT upon removal of $\mathrm{Mg}^{2+}-2$ (Table 1), which is consistent with the observation that the $\mathrm{Mg}^{2+}-2$ 
a

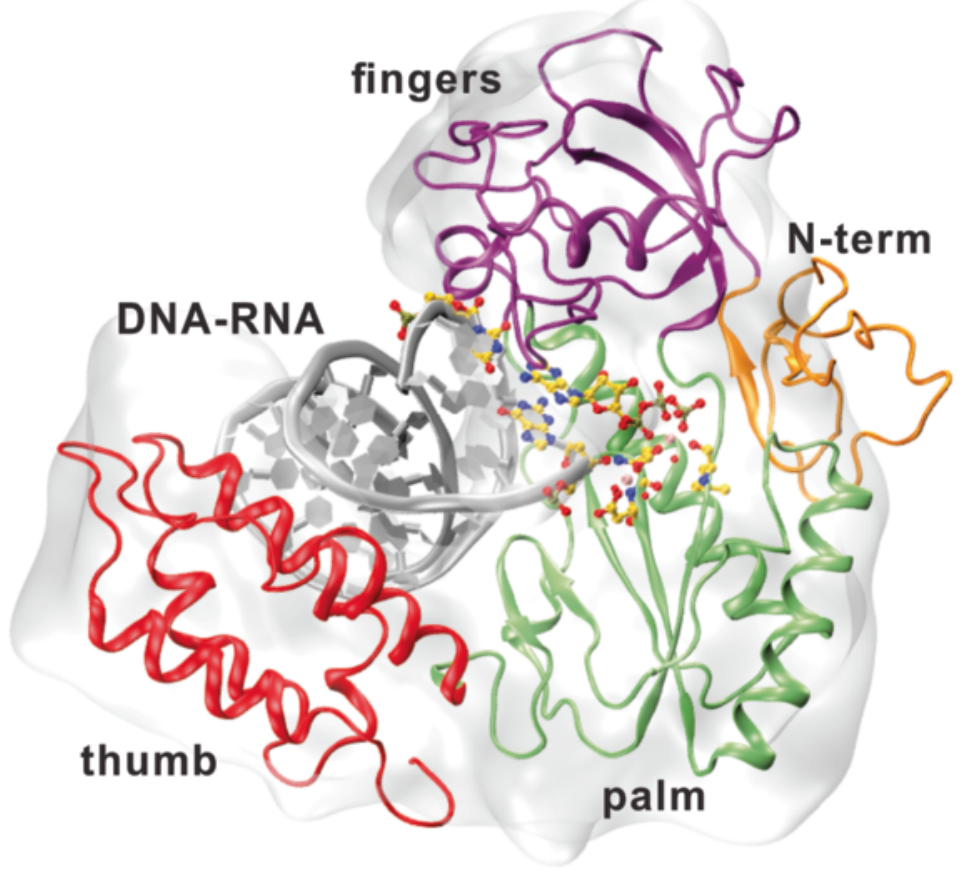

b

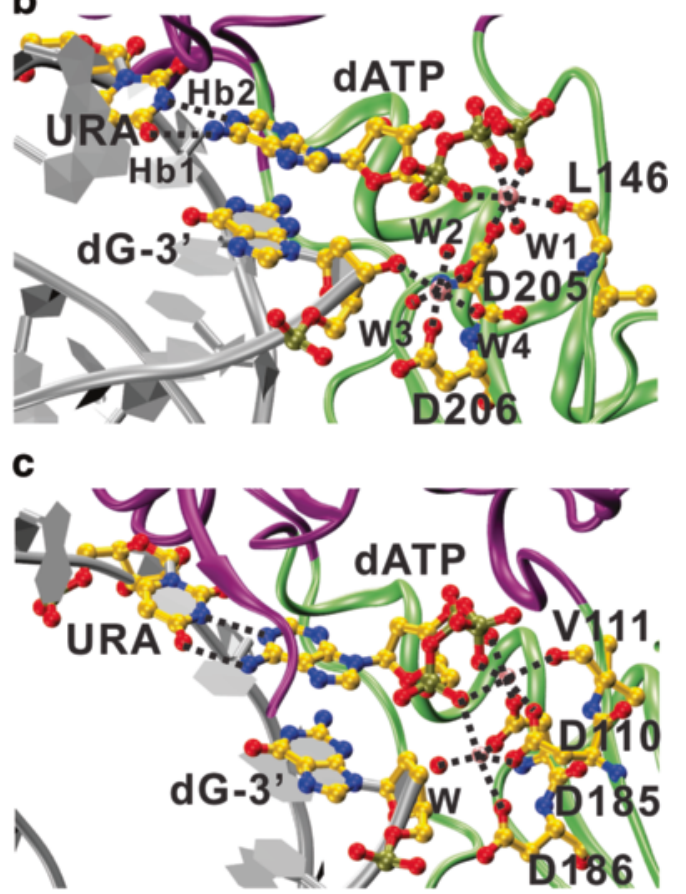

Fig. 2 The structure of HBV-RT4/DNA-RNA/dATP and the active site $\mathrm{Mg}^{2+}$ network in comparison with that from the HIV-RT/DNA-RNA/dATP crystal structure. a The HBV-RT4/DNA-RNAVdATP structure model is shown in cartoon with each domain in different color (N-terminal: orange; fingers: purple; thumb: red; palm: green; dsDNA: silver). The substrate dATP and the residues involved in direct interaction with it are in stick and ball representation. The corresponding part from the template HIV-RT/DNA-RNA/dATP (PDB code: 4PQU; chain A, residue 1 to residue 320) is shown in gray surface representation, structurally aligned to the HBV-RT4/DNA-RNA/dATP model; $\mathbf{b}$ The active site configuration of the HBV-RT4/DNA-RNA/ dATP model. The non-bonded interactions are explicitly represented as black dashed lines as the two H-bonds ( $\mathrm{Hb} 1 \mathrm{and} \mathrm{Hb2),} \mathrm{and} \mathrm{the} \mathrm{ionic}$ bonds formed between the $\mathrm{Mg}^{2+}$ ions (in red ball representation without labeling) and the chelating oxygen atoms. The water oxygen atoms participating in the chelation are labeled as from W1 to W4; c The active site configuration of the HIV-RT/DNA-RNA/dATP crystal structure, only one water molecule (labeled as W) is involved in the $\mathrm{Mg}^{2+}$ network. The D110 has no counterpart in the HBV-RT model. The hydrogen atoms are hided in the representations for clearance

is not directly involved in the substrate binding. On the other hand, it is noticeable that oxygen from the hydroxyl group of dG-3' is directly involved in the chelation of the $\mathrm{Mg}^{2+}-2$ throughout the simulation (Fig. 3b). Since the nature of the incorporation of a dNTP is to form a phosphodiester bond between the oxygen of the $3^{\prime}-\mathrm{OH}$ and the $\alpha-\mathrm{P}$ of the triphosphate group of the $\mathrm{dNTP}$, the network formed by the two $\mathrm{Mg}^{2+}$ ions is the optimal configuration for the impending chemical reaction. The distance between the oxygen of the 3'-OH and the $\alpha-\mathrm{P}$ of the triphosphate group of the dATP was also monitored for each of the HBV-RT variant over the simulations (data not shown). As further separation of the 3 '-OH and the $\alpha-\mathrm{P}$ did not appear in any of our $\mathrm{Mg}^{2+}-1$ only system, the $\mathrm{Mg}^{2+}$ network seems to contribute to the function of the enzyme in a way other than merely maintaining the distance between the 3'-OH of the extending DNA strand and the $\alpha-\mathrm{P}$ of the dATP. Presumably, forming a coordination bond with the $\mathrm{Mg}^{2+}-2$ would render the oxygen atom in the 3'-OH more favorable to initiation of the nucleophilic attack on the $\alpha-P$, thereby flavoring the transcriptase activity of the enzyme.

\section{Binding free energy of TFV-DP to HBV-RT}

Drug resistance often arises from structural alterations consequent to mutations in the target protein, which somehow disrupts or interferes with drug binding. To understand the underlying mechanism of the differential response to TDF treatment, we start by asking whether the TFV-DP binds differently to the RT variants from patients with slow or rapid response to TDF. In building a structural model of HBV-RT4/DNA-RNA/TFV-DP, we started with the HBV-RT4 model generated by ITASSER and AIDA (Additional file 2: Figure S2), then inserted the duplex DNA-RNA taken originally from the HIVRT/DNA-RNA/dATP template structure [22], and finally incorporated the TFV-DP from the HIV-RT/DNADNA/TFV-DP structure (PDB code: 1 T05) [19]. Models for the other four variants were built analogously from the homology models we obtained using the HBV-RT4 as template, into which the same DNA-RNA duplex and 

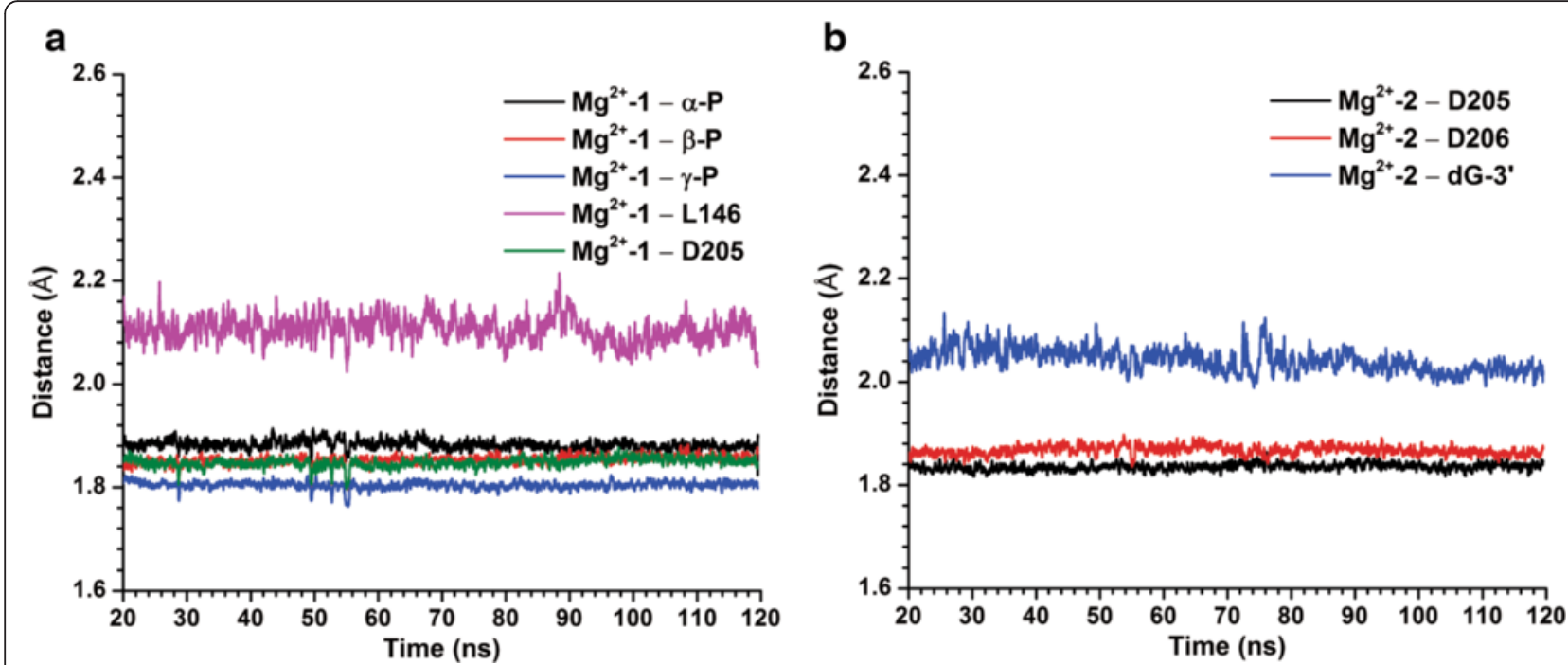

Fig. 3 Distances from the $\mathrm{Mg}^{2+}$ ions to non-water atoms which participating in its chelation. a The distance profile of the $\mathrm{Mg}^{2+}-1$ to the chelating atoms; b The distance profile of the $\mathrm{Mg}^{2+}-2$ to the chelating atoms. Each oxygen participating in the chelation is represented by the residue name

TFV-DP were incorporated. We then pursued MD simulations to refine all the HBV-RT/DNA-RNA/TFV-DP structures. The centroid of the major cluster from the second half of each MD trajectory was then chosen as representative model.

Here we present the HBV-RT4/DNA-RNA/TFV-DP model. As seen in Fig. 4a, the incorporation of the TFVDP did not elicit any disruption of the secondary structural elements of the HBV-RT4. The domain arrangement persists as they are in the HBV-RT4/DNA-RNA/dATP complex (Fig. 2a), indicating the model is in a proper functional state. At the active site, the TFV-DP associates with the enzyme in the exact fashion as we identified in the natural substrate-bound complex: the base-ring end of the TFV-DP is anchored by two persistent $\mathrm{H}$-bonds, and the triphosphate end of it is strongly coupled by the $\mathrm{Mg}^{2+}$ chelation network (Fig. 4b). While $\mathrm{Mg}^{2+}-2$ was missing in the HIV-RT/DNA-DNA/TFV-DP crystal structure (Fig. 4c), we believe that the integrity of the $\mathrm{Mg}^{2+}$ network is important for maintaining the proper configuration of the active site, as is seen in the HIV-RT/DNA-RNA/dATP crystal structure and in all of our HBV-RT/DNA-RNA/ dATP systems with two $\mathrm{Mg}^{2+}$ ions. Convinced of the potential catalytic role of the $\mathrm{Mg}^{2+}-2$, we opted to include the $\mathrm{Mg}^{2+}-2$ in all of our HBV-RT/DNA-DNA/TFV-DP systems. The result that the $\mathrm{Mg}^{2+}$ network is stable across all variants echoes the importance and validity of the addition.

We were then able to compute the binding energies of the dATP and TFV-DP to the HBV-RT/DNA-RNA complex with extensive sampling of the complex structures through MD simulations using the solvated interaction energies (SIE) method [24, 25]. The favorable and consistent binding energies of dATP to the variants observed in both $\mathrm{Mg}^{2+}$ systems (Table 1) indicate strong binding of the natural substrate to the enzyme, hence proper configuration of the active sites for all of our HBV-RT variant models. As for the TFV-DP ligand, the SIE results also show favorable binding to all HBV-RT variants (Table 1), with no substantial differences between them $\left(<1 \mathrm{Kcal} \mathrm{mol}^{-1}\right)$. These findings suggest that RT enzymatic activity and RT's affinity to bind TFV-DP are not altered by the amino-acid heterogeneity observed among the HBV-RT variants examined herein, which, taken together with the observed stability of ligandprotein interactions and consistency in the structural

Table 1 Approximated binding free energies with standard errors of dATP or TNV to HBV-RT/DNA-RNA using Solvated Interaction Energy method (SIE)

\begin{tabular}{lllll}
\hline Category & Variant & dATP, one $\mathrm{Mg}^{2+}\left(\mathrm{Kcal} \mathrm{mol}^{-1}\right)$ & $\mathrm{dATP}, \mathrm{two} \mathrm{Mg}^{2+}\left(\mathrm{Kcal} \mathrm{mol}^{-1}\right)$ & $\mathrm{TFV}_{-\mathrm{DP}, \mathrm{two} \mathrm{Mg}^{2+}\left(\mathrm{Kcal} \mathrm{mol}^{-1}\right)}$ \\
\hline RR & RT5 & $-10.43 \pm 0.02$ & $-9.93 \pm 0.02$ & $-8.91 \pm 0.01$ \\
& RT3 & $-11.51 \pm 0.02$ & $-11.21 \pm 0.03$ & $-9.22 \pm 0.01$ \\
SR & RT1 & $-10.59 \pm 0.01$ & $-9.69 \pm 0.02$ & $-9.56 \pm 0.01$ \\
& RT4 & $-12.51 \pm 0.01$ & $-9.50 \pm 0.02$ & $-9.05 \pm 0.02$ \\
& RT2 & $-10.51 \pm 0.02$ & $-10.59 \pm 0.01$ & $-9.51 \pm 0.01$ \\
\hline
\end{tabular}




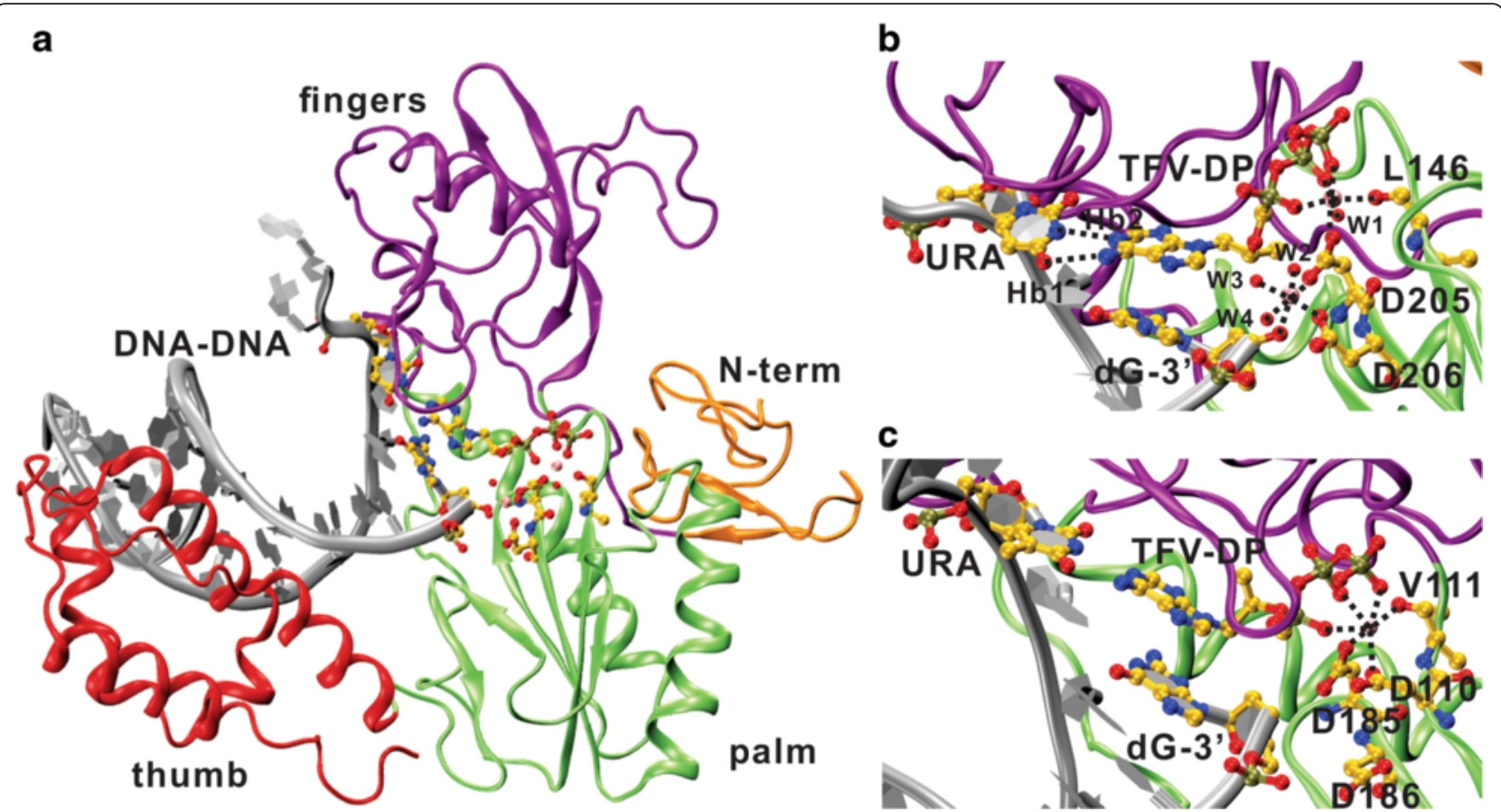

Fig. 4 The structure of HBV-RT4/DNA-RNA/TFV-DP and the active site $\mathrm{Mg}^{2+}$ network in comparison with that from the HIV-RT/DNA-DNATFV-DP crystal structure. a The HBV-RT4/DNA-RNA TFV-DP structure model is shown in cartoon with each domain in different color (N-terminal: orange; fingers: purple; thumb: red; palm: green; dsDNA: silver). The substrate TFV-DP and the residues involved in direct interaction with it are in stick and ball representation; $\mathbf{b}$ The active site configuration of the HBV-RT4/DNA-RNA/TFV-DP model. The non-bonded interactions are explicitly represented as black dashed lines as the two $\mathrm{H}$-bonds ( $\mathrm{Hb} 1$ and $\mathrm{Hb} 2$ ), and the ionic bonds formed between the $\mathrm{Mg}^{2+}$ ions (in red ball representation without labeling) and the chelating oxygen atoms. The water oxygen atoms participating in the chelation are labeled as from W1 to W4; c The active site configuration of the HIV-RT/DNA-DNA/TFV-DP crystal structure, only one $\mathrm{Mg}^{2+}$ is identified in the active site and no water molecule is involved. The hydrogen atoms are hided in the representations for clearance

configuration of the active site in all HBV-RT/DNARNA/TFV-DP complexes, suggest that factors other than a decreased binding affinity of HBV-RT to TFV-DP might be influencing therapy response in patients.

Interestingly, evidence of there being no substantial changes in drug-binding affinity in our HBV-RT/DNARNA/TFV-DP complexes (Table 1) is in agreement with our previous study [26]. A potential prediction model of response to TDF revealed that genetic diversity outside the RT domain of polymerase contributed robust predictive features strongly associated to SR/RR characteristics of patients, whereas in RT none were found [26]. Moreover, a switch in high-frequency intra-host HBV variants was observed in patient 4, which did not involve changes in RT. Amino-acid substitution S202P (GenBank reference AY AY233278) in the spacer domain of polymerase presented after 4 weeks of TDF treatment, suggesting a possible association between response to TDF and amino-acid heterogeneity in spacer, a suspected intrinsically disordered protein (IDP) [27]. In fact, there is compelling evidence of the involvement of IDPs in evolution of drug resistance in cancer cells [28, 29] and HBV [27]. Nevertheless, an association between amino-acid substitutions in HBV variants RT1-RT5 and differential response to TDF treatment cannot be dismissed solely based on findings presented herein. Drug-binding affinity may not be the only mechanism involved in response rates or resistance to TDF. Amino-acid substitutions observed in HBV-RT could be affecting some other structure-activity-resistance relationships. In addition, intramolecular conformational changes in RT and intermolecular dynamics of the RT complex provide an alternative mechanism for drug resistance to NNRTIs [30].

\section{Conclusion}

While many drug resistant mutants of HBV-RT have been identified, very little is known about the molecular mechanism driving resistance development. Compared to the massive structural and biochemical studies than have been conducted on HIV-RT drug resistant mutants, HBV-RT has not drawn much attention among structural biologists. However, over 350 million people have developed $\mathrm{CHB}$ and it remains a serious, global health problem [9]. Therefore, it is important to unravel the drug resistance associated with anti-viral treatment to HBV infections. 
To probe the structural basis for HBV-RT drug resistance, researchers rely on structural modeling approaches using crystal structures of HIV-RT as a starting point [17, 18, 31-33]. As protein structures are dynamic and strongly correlated with its functional states, we devised a novel hybrid approach in which we utilized the most relevant X-ray crystal structures (in terms of its functional state) to model the initial structures of HBV-RT and then extensively sampled the conformational space with experimentally derived constraints (crystal contacts between substrate and protein) to select the final representative models (Additional file 3: Figure S3). Without direct experimentally derived structure data on HBV-RT to test the models, this rigorous approach presumably generates structure models of the highest confidence and relevance to the particular biological function of interest. However, as this approach comprises only physics-based methods, it is advisable to apply other methods to evaluate the resulting model from a different perspective. In this study, we have applied a statistical potential based method (ProSAWeb) [34] to evaluate our model. The resulting Z-score for our HBV-RT4 model confirms good quality in terms of the naturalness defined by sets of X-ray and NMR structures (Additional file 4: Figure S4A), and the local quality plot indicates the majority of the HBV-RT4 model is of high quality measured by a knowledge-based energetic term (Additional file 4: Figure S4B).

Using these models to probe drug-protein interaction, we focused on the TFV-DP binding. The present findings indicate that, despite sequence changes in or around the NRTI binding site, favorable drug-protein interactions persisted. The dynamics of how key molecular groups interact, probed in our studies, reveal new insights into the mechanisms, through which HBV-RT couples to its natural substrate. However, this apparent drug-protein interaction stability leaves us without a good mechanistic rationalization as to why or how HBV-RT amino-acid polymorphisms may contribute to reduced rates of viral clearance responses to TDF treatment, as is often observed in SR patients.

The modeling approach presented in this study can be extended to other drugs and serve as a paradigm to ascertain drug binding and estimate binding free energies. It is important to note that the starting conformation (the binding site and the particular interaction pattern between the drug and protein) would have much effect on the results. Therefore, it is essential to start modeling using all available information for the accurate and reliable prediction of the binding site structure.

\section{Methods}

\section{Patients and HBV quasispecies (QS)}

Five patients from Study GS-US-203-0101, a phase 2 study evaluating TDF and FTC + TDF in treatment naive patients with HBV DNA $>1.7 \times 107 \mathrm{IU} / \mathrm{mL}$ and normal ALT levels for 192 weeks, were selected [35]. 3 patients had a slow response (SR), never achieving HBV DNA $<69 \mathrm{IU} / \mathrm{mL}$ through 192 weeks of treatment, while 2 patients had a rapid response (RR), achieving HBV DNA $<69 \mathrm{IU} / \mathrm{mL}$ by Week 96 . Patients were matched by HBV DNA, ALT, and HBeAg status at baseline. All patients received TDF monotherapy. Whole-genome sequences of HBV QS were obtained using end-point limitingdilution real-time PCR coupled with sequencing. Baseline (BL), Week 4 (W4), and Week 40 (W40) time points were evaluated for each patient. Median joining networks (MJN) were performed using Network 4.0 to analyze HBV QS genetic diversity [36]. The highfrequency variants of intra-host HBV QS from each patient were selected to predict the atomic structure of the RT protein domain in HBV polymerase (protein positions 349 - 693, based on GenBank reference sequence AY233278): from SR patients, variants RT1 and RT2 of HBV genotype C (GT C) and RT4 (GT B), and from RR patients, variants RT3 (GT C) and RT5 (GT B).

\section{Structural modeling of HBV-RT variants}

Due to the nonexistence of experimental 3D-structures of HBV-RT, a hybrid approach to protein 3D-strucutre prediction was implemented to generate accurate, atomistic structures of the NRTI binding site of HBV-RT. Three major steps were involved in the modeling. First, all the representative HBV-RT sequences were submitted to ITASSER web-server [20, 21], including specification on an homologous HIV-RT structure complex (PDB accession code: $4 \mathrm{PQU}$ ) as an additional template [20]. The model of the highest $\mathrm{C}$-score [21] were then chosen to proceed. The N-terminal 1-40 amino-acid of HBV-RT were modeled separately by ITASSER, due to a relatively low local accuracy for the initial model of the entire length. The $\mathrm{N}$-terminal structure was then assembled to the preliminary model by using $A b$ Initio Domain Assembly Server (AIDA) [23].

Second, all the HBV-RT variant models were then pooled for inspection to identify the one(s) with the folding pattern most consistent with that seen in the designated HIV-RT structure.

Last, variant HBV-RT4 was chosen for further refinement using multi-nanosecond molecular dynamics (MD) simulation with the native hybrid DNA-RNA duplex and with dATP incorporated into the active site, according to their original orientation in the HIV-RT/DNA-RNA/ dATP crystal structure [22]. Pairwise rmsd clustering analysis of the second half of the MD trajectory was then performed to select the centroid of the dominant cluster as the final model of HBV-RT4/DNA-RNA/dATP. The structures of the other variants were then obtained by homology modeling using Modeller 9.13 [37, 38] with 
the HBV-RT4 as template, followed by same MD simulation procedures, also with the native hybrid DNA-RNA duplexes and the dATPs superimposed to the active sites.

In the case of modeling the HBV-RT4/DNA-RNA/ TFV-DP variants, we replaced the dATP with TFV-DP in the initial conformation of the HBV-RT4/DNA-RNA/ dATP MD simulation, maintaining the crystal contacts between TFV-DP and corresponding residues that was identified from the HIV-RT4/DNA-DNA/TFV-DP crystal structure (PDB code: 1 T05) [19].

\section{Molecular dynamic (MD) simulation}

To conduct MD simulations, hydrogens were first added by the tleap module of AMBER 14 [39], and the ionizable side chains of the proteins were assigned to their ionization states at pH 7.0 using the WHATIF webserver [40]. Each system was then solvated with TIP3P water molecules [41] leaving a minimum distance of $10.0 \AA$ from the protein surface to the edge of the simulation box. Counter-ions were added to neutralize the net charge and reach $100 \mathrm{mM} \mathrm{NaCl}$ concentration to mimic physiological conditions. The systems were then minimized and equilibrated - with extra bonds constraints $\left(2.5 \mathrm{kcal} \mathrm{mol}^{-1} \AA^{-2}\right)$ on the $\mathrm{Mg}^{2+}$ ions and on the ionic bonded oxygen atoms - for 10-20 ns before free production runs in the isothermal isobaric ensemble $(1 \mathrm{~atm}, 300 \mathrm{~K})$. The short-range non-bonded interactions were evaluated by employing a cut off of $10 \AA$ with a switching function starting at $8.5 \AA$. The smooth particle mesh Ewald (SPME) algorithm were applied in computing the ong-range electrostatic interactions [42]. All the bonds formed by hydrogen and heavy atoms were fixed to eliminate the most frequent oscillatory motions. The $r-$ RESPA multiple time step method [43] was applied with a 2 fs time step for bonded, 2 fs for short-range non-bonded interactions and 4 fs for long-range electrostatic interactions. All simulations were performed using the NAMD 2.9 code $[44,45]$ with the AMBER Parm99 parameter set [46] containing the force field for nucleic acids and proteins. The parameterization of TFV-DP was conducted using Antechamber [47] with the general Amber force field (GAFF) [46, 48]. Data were analyzed using the CPPTRAJ utility in AMBER [49] and custom VMD TCL scripts [50]. Time evolution of the rmsd values for the HBV-RT in each simulation system was monitored for simulation convergence (Additional file 5: Figure S5).

\section{Binding free energy approximation}

To compute the binding free energy of dATP or TFVDP to the HBV-RT/DNA-RNA complex we employed the solvated interaction energies (SIE) method [24, 25], which is an end point method that shares elements from the linear interaction energy (LIE) approach [24, 25]. Frames at 20 ps intervals from the last 60 ns the MD trajectories (the rest of the trajectories were discarded as equilibration) were sampled for the SIE calculations. In total 3000 frames were used for averaging. The SIE estimates binding free energy as:

$$
\begin{aligned}
\Delta G_{\text {bind }}\left(\rho, D_{\text {in }}, \alpha, \gamma, C\right)= & \alpha \times\left[\Delta E_{v d W}+\Delta E_{\text {Coul }}\left(D_{\text {in }}\right)\right. \\
& \left.+\Delta G_{R F}\left(\rho, D_{\text {in }}\right)+\gamma \Delta S A(\rho)\right]+C
\end{aligned}
$$

where $\Delta E_{v d W}$ and $\Delta E_{C o u l}$ represents the intermolecular van der Waals and Coulomb interaction energy between protein and ligand, respectively. The values were computed using he AMBER ff99SB parameter set. $\Delta G_{R F}$ is the reaction field energy change upon ligand binding, computed by solving the Poisson equation with program BRI BEM [51], and using a molecular surface generated with a variable-radius solvent probe [52]. The $\triangle S A$ is the molecular surface area change upon ligand binding. $\rho$ is the linear scaling factor of the van der Waals radii for the AMBER99 force field; $D_{i n}$ is the dielectric constant for solute interior; $\gamma$ is the coefficient for computing the free energy associated with the surface area change upon binding; $\alpha$ is the pre-factor that quantifies the loss of configurationally entropy upon binding; $C$ is a constant that includes protein-dependent contributions not explicitly modeled by SIE. The parameters were fitted to the absolute binding free energies for a set of 99 protein-ligand complexes to obtain the optimal/default values of them as $\alpha=0.1048, D_{\text {in }}=2.25, \rho=1.1, \gamma=0.0129 \mathrm{Kcal} \mathrm{mol}^{-1} \AA^{-2}$, and $C=-2.89 \mathrm{Kcal} \mathrm{mol}^{-1}[24,25]$, which are also the values we used in our SIE calculations.

\section{Additional files}

Additional file 1: Figure S1. The initial structural models of HBV-RT variants obtained from ITASSER in comparison to the corresponding part in the template crystal structure: A) HBV-RT5; B) HBV-RT1; C) HBV-RT4; D) HBV-RT2; E) HBV-RT3; F) Residues 1 to 320 of the HIV-RT/DNA-RNA/dATP crystal structure (PDB code: $4 \mathrm{PQU}$ ). The 40 aa N-terminal of the HBV-RT structure are shown in orange, the hairpin structural element in the fingers domain are in red, and the rest of the structure are in cyan. (TIF 5479 kb)

Additional file 2: Figure S2. The structure models of HBV-RT4 obtained from ITASSER followed by ab initio assembly using AIDA. The 40 aa $\mathrm{N}$-terminal of the HBV-RT structure is shown in orange, the hairpin structural element in the fingers domain in red, and the rest of the structure in cyan. (TIF 5196 kb)

Additional file 3: Figure S3. The schematic plot of the hybrid modeling approach used in HBV-RT/DNA-RNA/dATP and in HBV-RT/DNARNA/TFV-DP modeling. (TIF $2413 \mathrm{~kb}$ )

Additional file 4: Figure S4. Quality of HBV-RT4 model evaluated by statistical potential: A) Overall quality score of the HBV-RT4 model (black dot): Z-score $=-6.34$; B) Local model quality scores as knowledge-based energies versus residue position, with positive values indicate possible problematic or erroneous parts in the model. (TIF $2605 \mathrm{~kb}$ )

Additional file 5: Figure S5. RMSD evolution as a function of time for all the MD simulations: A) HBV-RT/DNA-RNA/dATP with $\mathrm{Mg}^{2+}-1$ only in the active site; B) HBV-RT/DNA-RNA/dATP with two $\mathrm{Mg}^{2+}$ ions in the active site; C) HBV-RT/DNA-RNA/TFV-DP with two $\mathrm{Mg}^{2+}$ ions in the active site. (TIF 2569 kb) 


\section{Abbreviations}

CHB, chronic hepatitis $B$; dATP, deoxyadenosine triphosphate; DNA, deoxyribonucleic acid; ds-DNA, double stranded DNA; HBV, hepatitis B virus; MD, molecular dynamics; NNRTI, non-nucleoside reverse transcriptase inhibitors; NRTIs, nucleoside and nucleotide reverse transcriptase inhibitors; rmsd, root mean square deviation; $R N A$, ribonucleic acid; $R R$, rapid response; $R T$, reverse transcriptase; SIE, solvated interaction energy; SR, slow response; TDF, tenofovir disoproxil fumarate; TFV-DP, tenofovir diphosphate (active form of TDF)

\section{Acknowledgements}

We thank Dr. Formijn J van Hemert from University of Amsterdam and Dr. Jianyi Yang from University of Michigan for advises on modeling the HBV-RT structure. We thank Dr. Robert Wohlhueter (Georgia State University) and Dr. Michael Mitchell (Gilead Science, Inc.) for their contribution in editing the manuscript.

\section{Declarations}

This article has been published as part of BMC Bioinformatics Volume 17 Supplement 8, 2016. Selected articles from the 11th International Symposium on Bioinformatics Research and Applications (ISBRA '15): bioinformatics. The full contents of the supplement are available online https://bmcbioinfor matics.biomedcentral.com/articles/supplements/volume-17-supplement-8.

\section{Funding}

This study was supported by CDC intramural funding and by APHL postdoctoral fellowship funding (2014-2015) to [X.X]. This information is distributed solely for the purpose of pre-dissemination peer review under applicable information quality guidelines. It has not been formally disseminated by the Centers for Disease Control and Prevention/Agency for Toxic Substances and Disease Registry. It does not represent and should not be construed to represent any agency determination or policy. Publication costs for this work were funded by CDC

\section{Availability of data and materials}

Data and materials are available upon request.

\section{Authors' contributions}

$X X, Y K$ and $J L$ designed the experiment; XX implemented the 3D modeling and MD simulations, analyzed the 3D output data and prepared the manuscript; HT and LG-R conducted DNA sequencing of HBV variants; GX conducted clean up and formatting of DNA sequence data; KK, AG and MP provided serum samples and patient clinical/demographic information. All authors discussed the results and implications and commented on the manuscript at all stages.

\section{Competing interests}

X.X., H.T., G.X., L.G-R., Y.K. and J.L. declare no competing interests. At the time the work was completed, K.K., A.G., and M.P. were employees and stockholders of Gilead Sciences.

\section{Consent for publication}

Not applicable.

\section{Ethics approval and consent to participate}

All patients provided written consent, and the clinical trial protocol was approved by the Institutional Review Boards of each participating institution.

\section{Author details}

'Division of Viral Hepatitis, National Center for HIV, Hepatitis, STD and TB Prevention, Centers for Disease Control and Prevention, Atlanta, GA 30333, USA. ${ }^{2}$ Gilead Sciences, Inc., Foster City, CA, USA.

\section{Published: 31 August 2016}

\section{References}

1. Menendez-Arias L, Alvarez M, Pacheco B. Nucleoside/nucleotide analog inhibitors of hepatitis B virus polymerase: mechanism of action and resistance. Curr Opin Virol. 2014;8:1-9.

2. Coppolino $\mathrm{G}$ et al. The case of chronic hepatitis $B$ treatment with tenofovir: an update for nephrologists. J Nephrol. 2015;28(4):393-402.
3. Arimilli MN et al. Synthesis, in vitro biological evaluation and oral bioavailability of 9-[2-(phosphonomethoxy)propyl]adenine (PMPA) prodrugs. Antivir Chem Chemother. 1997;8(6):557-64.

4. Robbins BL et al. Anti-human immunodeficiency virus activity and cellular metabolism of a potential prodrug of the acyclic nucleoside phosphonate 9-R-(2phosphonomethoxypropyl)adenine (PMPA), Bis(isopropyloxymethylcarbonyl) PMPA. Antimicrob Agents Chemother. 1998;42(3):612-7.

5. Miller MD et al. Antiviral activity of tenofovir (PMPA) against nucleosideresistant clinical HIV samples. Nucleosides Nucleotides Nucleic Acids. 2001;20(4-7):1025-8.

6. Durand-Gasselin $L$ et al. Nucleotide analogue prodrug tenofovir disoproxil enhances lymphoid cell loading following oral administration in monkeys. Mol Pharm. 2009;6(4):1145-51.

7. Perry CM, Simpson D. Tenofovir disoproxil fumarate: in chronic hepatitis B. Drugs. 2009:69(16):2245-56.

8. Michailidis E et al. Antiviral therapies: focus on hepatitis B reverse transcriptase. Int J Biochem Cell Biol. 2012:44(7):1060-71.

9. Jones $\mathrm{SA}, \mathrm{Hu}$ J. Hepatitis B virus reverse transcriptase: diverse functions as classical and emerging targets for antiviral intervention. Emerg Microbes Infect. 2013;2:e56

10. Jung SK et al. Tenofovir disoproxil fumarate monotherapy for nucleos(t)ide analogue-naive and nucleos(t)ide analogue-experienced chronic hepatitis B patients. Clin Mol Hepatol. 2015;21(1):41-8.

11. Kitrinos $\mathrm{KM}$ et al. No detectable resistance to tenofovir disoproxil fumarate after 6 years of therapy in patients with chronic hepatitis B. Hepatology. 2014;59(2):434-42.

12. Marcellin $P$, Asselah T. Long-term therapy for chronic hepatitis B: hepatitis $B$ virus DNA suppression leading to cirrhosis reversal. J Gastroenterol Hepatol. 2013;28(6):912-23.

13. Baran B et al. Efficacy of tenofovir in patients with Lamivudine failure is not different from that in nucleoside/nucleotide analogue-naive patients with chronic hepatitis B. Antimicrob Agents Chemother. 2013;57(4):1790-6.

14. Das $\mathrm{K}$ et al. Structural basis for the role of the K65R mutation in HIV-1 reverse transcriptase polymerization, excision antagonism, and tenofovir resistance. J Biol Chem. 2009;284(50):35092-100

15. Hachiya $A$ et al. K70Q adds high-level tenofovir resistance to "O151M Complex" HIV reverse transcriptase through the enhanced discrimination mechanism. PLoS One. 2011;6(1):e16242.

16. White $\mathrm{KL}$ et al. Molecular mechanisms of tenofovir resistance conferred by human immunodeficiency virus type 1 reverse transcriptase containing a diserine insertion after residue 69 and multiple thymidine analog-associated mutations. Antimicrob Agents Chemother. 2004;48(3):992-1003.

17. van Hemert FJ, Berkhout B, Zaaijer HL. Differential binding of tenofovir and adefovir to reverse transcriptase of hepatitis B virus. PLoS One. 2014:9(9):e106324.

18. Das K et al. Molecular modeling and biochemical characterization reveal the mechanism of hepatitis B virus polymerase resistance to lamivudine (3TC) and emtricitabine (FTC). J Virol. 2001;75(10):4771-9.

19. Tuske $\mathrm{S}$ et al. Structures of HIV-1 RT-DNA complexes before and after incorporation of the anti-AIDS drug tenofovir. Nat Struct Mol Biol. 2004;11(5):469-74.

20. Zhang Y. I-TASSER server for protein 3D structure prediction. BMC Bioinformatics. 2008:9:40.

21. Roy A, Kucukural A, Zhang Y. I-TASSER: a unified platform for automated protein structure and function prediction. Nat Protoc. 2010;5(4):725-38.

22. Das $K$ et al. Structures of HIV-1 RT-RNA/DNA ternary complexes with dATP and nevirapine reveal conformational flexibility of RNA/DNA: insights into requirements for RNase H cleavage. Nucleic Acids Res. 2014;42(12):8125-37.

23. Xu D et al. AIDA: ab initio domain assembly server. Nucleic Acids Res. 2014:42(Web Server issue):W308-13.

24. Naim M et al. Solvated interaction energy (SIE) for scoring protein-ligand binding affinities. 1. Exploring the parameter space. J Chem Inf Model. 2007:47(1):122-33

25. Sulea T, Cui Q, Purisima EO. Solvated interaction energy (SIE) for scoring protein-ligand binding affinities. 2. Benchmark in the CSAR-2010 scoring exercise. J Chem Inf Model. 2011:51(9):2066-81.

26. Lara, J., et al., P0633 : Association of hepatitis B virus genetic diversity with tenofovir disoproxil fumarate response rates in immune tolerant chronic hepatitis B patients. Journal of Hepatology. 2015;62: p. S556.

27. Campo DS et al. Coordinated evolution of the hepatitis $B$ virus polymerase. In Silico Biol. 2011;11(5-6):175-82. 
28. Eldar A, Elowitz MB. Functional roles for noise in genetic circuits. Nature 2010;467(7312):167-73.

29. Sharma, S.V., et al., A Chromatin-Mediated Reversible Drug-Tolerant State in Cancer Cell Subpopulations. Cell. 2010;141(1): p. 69-80.

30. Schauer GD et al. Mechanism of allosteric inhibition of HIV-1 reverse transcriptase revealed by single-molecule and ensemble fluorescence. Nucleic Acids Res. 2014;42(18):11687-96.

31. Daga PR, Duan J, Doerksen RJ. Computational model of hepatitis B virus DNA polymerase: molecular dynamics and docking to understand resistant mutations. Protein Sci. 2010;19(4):796-807.

32. Walsh AW et al. Mechanistic characterization and molecular modeling of hepatitis B virus polymerase resistance to entecavir. PLoS One. 2010:5(2):e9195.

33. Lin $\mathrm{X}$ et al. A single amino acid in the reverse transcriptase domain of hepatitis B virus affects virus replication efficiency. J Virol. 2001;75(23):11827-33.

34. Wiederstein M, Sippl MJ. ProSA-web: interactive web service for the recognition of errors in three-dimensional structures of proteins. Nucleic Acids Res. 2007;35(Web Server issue):W407-10.

35. Chan HL et al. Effects of tenofovir disoproxil fumarate in hepatitis B e antigen-positive patients with normal levels of alanine aminotransferase and high levels of hepatitis B virus DNA. Gastroenterology. 2014;146(5):1240-8.

36. Bandelt HJ, Forster P, Rohl A. Median-joining networks for inferring intraspecific phylogenies. Mol Biol Evol. 1999;16(1):37-48

37. Eswar $\mathrm{N}$ et al. Comparative protein structure modeling using Modeller. Curr Protoc Bioinformatics. 2006;Chapter 5:Unit 56

38. Webb B, Sali A. Comparative protein structure modeling using MODELLER. Curr Protoc Bioinformatics. 2014;47:5 6 1-5 632

39. Case DA et al. The Amber biomolecular simulation programs. J Comput Chem. 2005;26(16):1668-88.

40. Vriend G. WHAT IF: a molecular modeling and drug design program. J Mol Graph. 1990;8(1):52-6. 29.

41. Jorgensen WL et al. Comparison of simple potential functions for simulating liquid water. J Chem Phys. 1983;79(2):10.

42. Essmann $U$ et al. A smooth particle mesh Ewald method. J Chem Phys. 1995;103:8577-93

43. Tuckerman M, Berne BJ, Martyna GJ. Reversible multiple time scale molecular-dynamics. J Chem Phys. 1992;97(3):1990-2001.

44. Kale $L$ et al. NAMD2: Greater scalability for parallel molecular dynamics. J Comput Phys. 1999;151(1):283-312.

45. Phillips JC et al. Scalable molecular dynamics with NAMD. J Comput Chem. 2005:26:1781-802.

46. Wang $\mathrm{J}$ et al. Development and testing of a general amber force field. J Comput Chem. 2004;25(9):1157-74.

47. Wang $\mathrm{J}$ et al. Automatic atom type and bond type perception in molecular mechanical calculations. J Mol Graph Model. 2006;25(2):247-60.

48. Hornak $V$ et al. Comparison of multiple Amber force fields and development of improved protein backbone parameters. Proteins. 2006;65(3):712-25.

49. Roe DR, Cheatham TE. PTRAJ and CPPTRAJ: software for processing and analysis of molecular dynamics trajectory data. J Chem Theory Comput. 2013:9(7):3084-95.

50. Humphrey W, Dalke A, Schulten K. VMD: visual molecular dynamics. J Mol Graph Model. 1996;14(1):33-8.

51. Aqvist J, Medina C, Samuelsson JE. A new method for predicting binding affinity in computer-aided drug design. Protein Eng. 1994; (3):385-91.

52. Jones-Hertzog DK, Jorgensen WL. Binding affinities for sulfonamide inhibitors with Human Thrombin Using Monte Carlo Simulations with a linear response method. J Med Chem. 1997:40(10):1539-49.

\section{Submit your next manuscript to BioMed Central and we will help you at every step:}

- We accept pre-submission inquiries

- Our selector tool helps you to find the most relevant journal

- We provide round the clock customer support

- Convenient online submission

- Thorough peer review

- Inclusion in PubMed and all major indexing services

- Maximum visibility for your research

Submit your manuscript at www.biomedcentral.com/submit
Biomed Central 